\title{
Plasticity of motor function and surgical outcomes in patients with cerebral arteriovenous malformation involving primary motor area: insight from fMRI and DTI
}

Lijun Wang ${ }^{1,2}$, Fuxin Lin ${ }^{1}$, Jun Wu1, Yuming Jiao ${ }^{1}$, Yong Cao ${ }^{1}$, Yuanli Zhao ${ }^{1}$ and Shuo Wang ${ }^{1 *}$

\begin{abstract}
Background: Patients who have a cerebral arteriovenous malformation (CAVMs) in the motor cortex can have displaced function. The finding and its relationship to recovery from surgery is not known.

Methods: We present the five cases with CAVMs involving precentral knob and/or paracentral lobule and without preoperative motor deficits. We used motor activation areas derived from Functional functional MRI (fMRI) as a region of interesting (ROI) to launch the plasticity of cerebrospinal tracts (CST). All the results were incorporated into the neuronavigation platform for surgical treatment. Intraoperative electric cortical stimulation (ECS) was used to map motor areas. Modified Rankin Scale (mRS) of hands and feets were performed on postoperative day 2, 7 and at month 3, 6 during follow-up period. All the patients suffered from motor deficits regardless of cortical activation patterns.
\end{abstract}

Results: Three patients showed functionally seeded CST in or around the AVM, and were validated by intraoperative electrical stimulation (ECS). Patient 4 had two aberrant functionally seeded fiber tracts away from the lesion, but were proved to be non-functional by postoperative motor deficits. Patient 3 with motor cortex and fiber tract within a diffuse AVMs nidus, complete paralysis of upper extremity after operation and has a persistent motor deficit during 6-month follow-up period.

Conclusions: The plasticity of motor cortex on fMRI doesn't prevent post-operative motor deficits. Functionally mapped fiber tract within or abutting AVM nidus predicts transient and persistent motor deficit.

Keywords: Cerebral arteriovenous malformations, Functional MRI, Diffusion tensor imaging, Plasticity, Primary motor area, Electric cortical stimulation

\section{Background}

Although cerebral arteriovenous malformations (cAVMs) may grow in eloquent areas such as the motor cortex, patients with a cAVMs usually do not present with neurological deficits unless it has ruptured. It has been postulated that when these lesions develop in the usual anatomical sites of eloquent cortex, neuroplasticity

\footnotetext{
* Correspondence: captainwang9858@126.com

1 Department of Neurosurgery, Beijing Tiantan Hospital, Capital Medical University, Beijing, P. R. China

Full list of author information is available at the end of the article
}

will result in cortical reorganization of the functional areas, with displacement to other regions. Therefore, function will not be impaired. Functional MRI (fMRI) [1, 2] and diffusion tensor imaging (DTI) [3-5] can detect plasticity of the brain cortex and fiber tracts in cAVMs patients. Currently, have seldom studies about fMRI and DTI to explore the relationship between the plasticity of motor cortex/fiber and surgical outcomes in patients of cAVMs involving primary motor area (PMA). We focused on intraoperative ECS to validate plasticity of motor cortex/fiber and determine the correlation of 
plasticity and postoperative motor deficits,to find evidence for patient selection before surgical treatment.

\section{Methods \\ Data collection and definition}

Between September 2013 and September 2014, five patients with AVMs involving primary and secondary motor cortex were reviewed from our AVMs database of a prospectively designed clinical trial (ClinicalTrials.gov Identifier: NCT01758211). In this study, all procedures were approved by the Institutional Review Board of Beijing Tiantan Hospital Affiliated to Capital Medical University, and informed written consent was obtained from all of the adult patients and from the parents of the pediatric patients. This consent was for both participation in the study and the publication of patient data. Two experienced neurosurgeons performed manual muscle strength testing of hands and feet by using the medical research council scale of $0-5$ in all of the patients and collected the other clinical information (FXL, JW) (Table 1). For transient deficits analysis, motor functions were evaluated on postoperative day 2 and 7. For persistent deficits analysis, motor deficits were evaluated at postoperative months 3 and 6 during follow-up period.

\section{Imaging}

MRI scan was performed on patients within 1 week before surgery on a 3.0 T MR system (SIEMENS Trio). The scan protocol includes: T1 anatomical image, blood oxygen level dependent fMRI (BOLD-fMRI), DTI and time-of-flight magnetic resonance angiography (TOFMRA). The sagittal T1 anatomy image was acquired by gradient-echo sequence, TR $2300 \mathrm{~m}$, TE $2.98 \mathrm{~ms}$, slice thickness $1 \mathrm{~mm}$, slices 176, FOV $256 \mathrm{~mm}$, flip angle $9^{\circ}$, matrix $64 \times 64$, voxel size $1 \times 1 \times 1 \mathrm{~mm}^{3}$, bandwidth 240. The BOLD-fMRI was collected with standard SP echo-planar imaging (EPI) measurement, TR 3000 ms,
TE $30 \mathrm{~ms}$, matrix $64 \times 64,30$ axial slices including all cerebral area, isotropic resolution of $3 \mathrm{~mm}$. The finger tap and foot flexion-extension movements were used as the motor stimulation paradigms for upper and lower limbs respectively, each with 64 repetitions, 24 s task and $24 \mathrm{~s}$ control state alternately. The DTI was acquired by DW-EPI technique: RT $6100 \mathrm{~ms}$, TE $93 \mathrm{~ms}$, slice thickness $3 \mathrm{~mm}$, slices 45 , FOV $230 \times 230 \mathrm{~mm}^{2}$, matrix $128 \times 128$ with a motion-probing gradient in 30 orientations. Axial TOF-MRA was acquired using a 3D TOF gradient-echo acquisition sequence RT $22 \mathrm{~ms}$, TE 3.86 ms, slice thickness $1 \mathrm{~mm}$, slices $36 \times 4$, FOV $220 \times 220$ $\mathrm{mm}^{2}$, Flip Angle $120^{\circ}$, Matrix $512 \times 512$. Finally, a significance threshold of $P<0.001$ was considered for identification of activated clusters. The anatomic locations of the activation and peak motor activity point for each paradigm were documented by two neuroradiologists. The activated areas were created as 3D objects to track fiber tracts. We used motor activation areas derived from fMRI as one principle ROI to launch CST, and the other ROI was located at the anterior-low-potion of pons (including both sides). The locations of the ROIs and the information about the tracked fiber tracts were also documented by the two neuroradiologists with consensus (Table 2).

Image sets acquired were processed on the iPlan 3.0 workstation (Brainlab, Heimstetten, Germany). All the image sets were automatically co-registered with each other and fused to the anatomical images by an automatic rigid registration. Finally, all the results were incorporated into the neuronavigation platform for surgical treatment.

\section{Surgery}

The operation was performed and was supervised by the experienced vascular neurosurgeon (SW). The neuronavigation system was used for image guidance during the surgery. All the patients underwent a craniotomy under

Table 1 Clinical characteristics of the patients and arteriovenous malformations (AVMs)

\begin{tabular}{|c|c|c|c|c|c|c|c|c|}
\hline $\begin{array}{l}\text { Patient } \\
\text { No. }\end{array}$ & Sex & $\begin{array}{l}\text { Age } \\
y\end{array}$ & Clinical presentation & Anatomic location of AVMs & $\begin{array}{l}\text { Martin-Spetzler } \\
\text { score }\end{array}$ & Nidus & $\begin{array}{l}\text { Pre-op muscle } \\
\text { strength }\end{array}$ & $\begin{array}{l}\text { Hemorrhage } \\
\text { history }\end{array}$ \\
\hline 1 & $\mathrm{~F}$ & 13 & Epilepsy & $\begin{array}{l}\mathrm{L} \text { anterior motor area, precentral } \\
\text { knob }\end{array}$ & S1V0E1 G2 & compact & V & $\mathrm{N}$ \\
\hline 2 & $\mathrm{~F}$ & 18 & $\begin{array}{l}\text { Numbness of right } \\
\text { limbs }\end{array}$ & L paracentral lobule & S1V0E1 G2 & compact & V & Y \\
\hline 3 & $\mathrm{~F}$ & 13 & Headache & $\begin{array}{l}\text { L superior parietal lobule, } \\
\text { precentral knob }\end{array}$ & S3V0E1 G4 & diffuse & V & $\mathrm{N}$ \\
\hline 4 & M & 33 & Headache & $\begin{array}{l}\text { R anterior motor area, precentral } \\
\text { knob }\end{array}$ & S2V0E1 G3 & compact & V & $\mathrm{N}$ \\
\hline 5 & $\mathrm{~F}$ & 18 & $\begin{array}{l}\text { Epilepsy of the } \\
\text { limbs }\end{array}$ & $\begin{array}{l}\text { R anterior motor area, precentral } \\
\text { knob }\end{array}$ & S2V0E1 G3 & compact & V & $\mathrm{N}$ \\
\hline
\end{tabular}

$L$ left, $R$ right, $S$ size of nidus, $V$ venous drainage, $E$ eloquence of adjacent brain, Pre-op preoperative. The muscle strength was scored according to the medical research council scale of $0-\mathrm{V}$ as follows: 0 , Flaccid; I, Only trace muscle contraction; II, Able to accomplish a full range of motion with gravity eliminated; III, Able to accomplish a full range of motion against gravity only; IV, Able to overcome moderate resistance; and V, Normal strength 
Table 2 Functional imaging characteristics and postoperative motor function

\begin{tabular}{|c|c|c|c|c|c|c|c|c|c|c|}
\hline \multirow[t]{2}{*}{$\begin{array}{l}\text { Patient } \\
\text { No. }\end{array}$} & \multicolumn{2}{|c|}{ BOLD activation areas } & \multicolumn{2}{|c|}{ CST derived from } & \multicolumn{2}{|c|}{$\begin{array}{l}\text { Stimulation positive } \\
\text { sites }\end{array}$} & \multicolumn{4}{|c|}{ Post-operative motor function } \\
\hline & $\begin{array}{l}\text { Contralesional } \\
\text { hemisphere }\end{array}$ & $\begin{array}{l}\text { Ipsilesional } \\
\text { hemisphere }\end{array}$ & $\begin{array}{l}\text { Contralesional } \\
\text { hemisphere }\end{array}$ & $\begin{array}{l}\text { Ipsilesional } \\
\text { hemisphere }\end{array}$ & cortex & $\overline{\text { subcortex }}$ & $\begin{array}{l}2^{\text {nd }} \\
\text { day }\end{array}$ & $\begin{array}{l}7^{\text {th }} \\
\text { day }\end{array}$ & $\begin{array}{l}3^{\text {rd }} \\
\text { month }\end{array}$ & $\begin{array}{l}6^{\text {th }} \\
\text { month }\end{array}$ \\
\hline 1 & None & $\mathrm{PMA}^{*}, \mathrm{SA}$ & None & PMA, SA & $\begin{array}{l}\text { Behind } \\
\text { lesion }\end{array}$ & $\begin{array}{l}\text { Behind } \\
\text { lesion }\end{array}$ & III & IV & V & V \\
\hline 2 & None & PMA, SMA* & None & SMA & None & $\begin{array}{l}\text { Before } \\
\text { lesion }\end{array}$ & IV & $\mathrm{IV}^{+}$ & V & V \\
\hline 3 & None & $\mathrm{PMA}^{*}$ & None & PMA & In lesion & In lesion & I & । & III & IV \\
\hline 4 & SPL, AMA & $\mathrm{SA}, \mathrm{SPL}^{*}$ & AMA & SPL & $\begin{array}{l}\text { Behind } \\
\text { lesion }\end{array}$ & $\begin{array}{l}\text { Behind } \\
\text { lesion }\end{array}$ & III & । & $U$ & $U$ \\
\hline 5 & $\mathrm{SPL}, \mathrm{PMA}^{*}$ & SA & none & SA & $\begin{array}{l}\text { Behind } \\
\text { lesion }\end{array}$ & $\begin{array}{l}\text { Behind } \\
\text { lesion }\end{array}$ & III & III & IV & IV \\
\hline
\end{tabular}

BOLD activation areas: BOLD activation areas elicited by contralesional hand or foot movement. Stimulation positive sites: Positive sites of electrical stimulation. Post-operative motor deficit: muscle strength of hand and/or foot contralateral to lesion hemisphere

PMA primary motor area, SMA sensorimotor area, SA sensory area, SPL superior parietal lobule, AMA anterior motor area, U: unavailable

* labeled the peak motor activity point

general anesthesia without the use of muscle relaxants. The AVMs resection was completed, and special attention was paid to preserve the functional cortex and the white matter tracts by subcortical stimulation and CST tractography, which was incorporated into the neuronavigation system. Electric stimulation of the cortex and subcortex (5-pulse sequence train; duration, 1 millisecond; $500 \mathrm{~Hz} ; 2 \mathrm{~mA}$ starting, stepwise increase of stimulation intensity up to $16 \mathrm{~mA}$ ), stimulating area stay a bit dry. Motor evoked potentials (MEPs) variation was recorded. Intraoperative ultrasound was used to detect residuals after the nidus was removed. The consistency between preoperative functional mapping and intraoperative electrophysiological examination was recorded by the operators. All the patients underwent CTA or DSA on the 3rd days after surgery. No residual cAVMs were found.

\section{Results}

Preoperative functional mapping was not consistent with intraoperative ECS results in case1, who had a cAVMs occupying the right percentral knob. Left hand movement elicited the sensory area (SA), superior parietal lobule (SPL) in the ipsilesional hemisphere and anterior motor area (AMA), SPL in the contralesional hemisphere. Two aberrant fiber tracts were tracked using the motor activation areas as the ROI. One derived from ipsilesional SPL, and the other one derived from contralateral AMA. Both of the two fiber tracts were not consistent with anatomic CST pathway and far away from the lesion (Fig. 1). But intraoperative ECS results showed that the positive stimulation sites were found at cortex and subcortex levels just posteriorthe lesion. Furthermore, the patient suffered from postoperative motor deficits, which confirmed the electrophysiological findings. In the other patients, the preoperative functional mappings were consistent with intraoperative ECS results. In case 2, a compact AVMs partially involved the left precentral knob. The residual precentral knob and SA were activated during right hand movement. Functionally tracked fiber tract was behind the lesion and located in a normal anatomic pathway (Fig. 2). Patient 3 possessed a partially aberrant fiber tract. In this patient, the lesion occupied the posterior part of left paracentral lobule. The activation areas elicited by right foot movement reorganized obviously at the anterior part of the paracentral lobule and supplementary motor area (SMA) compared with that in contralateral hemisphere (Fig. 3). The CST fiber tract in ipsilesional hemisphere originated from anterior part of the paracentral lobule and SMA then traveled backwards to the anatomical CST pathway, where the fiber was close to the lesion. The preoperative functional findings were validated by intraoperative electrophysiological results with no cortical positive stimulation point around the lesion and low amplitude subcortical positive stimulation points location in anterior of the lesion. Patient 4 had a diffuse nidus of AVMs, which involved left superior parietal lobule and precentral knob area. The only activation area elicited by right hand movement was within the nidus. The CST fiber tract originated from the activation area and then passed through the nidus (Fig. 4). Intraoperative ECS results were consistent with preoperative functional mapping as cortical and subcortical positive simulation points were found in the brain parenchyma. Patient 5 presented with epilepsy of bilateral hands caused by a AVMs involving the right precentral knob. Ipsilesional SA, contralesional SPL and contralesional primary motor area (PMA) were activated by left hand movement. The peak motor activity point was located in contralateral PMA. Functionally tracked fiber tracts derived from ipsilesional SA and travelled along the anatomic CST pathways. Interestingly no 


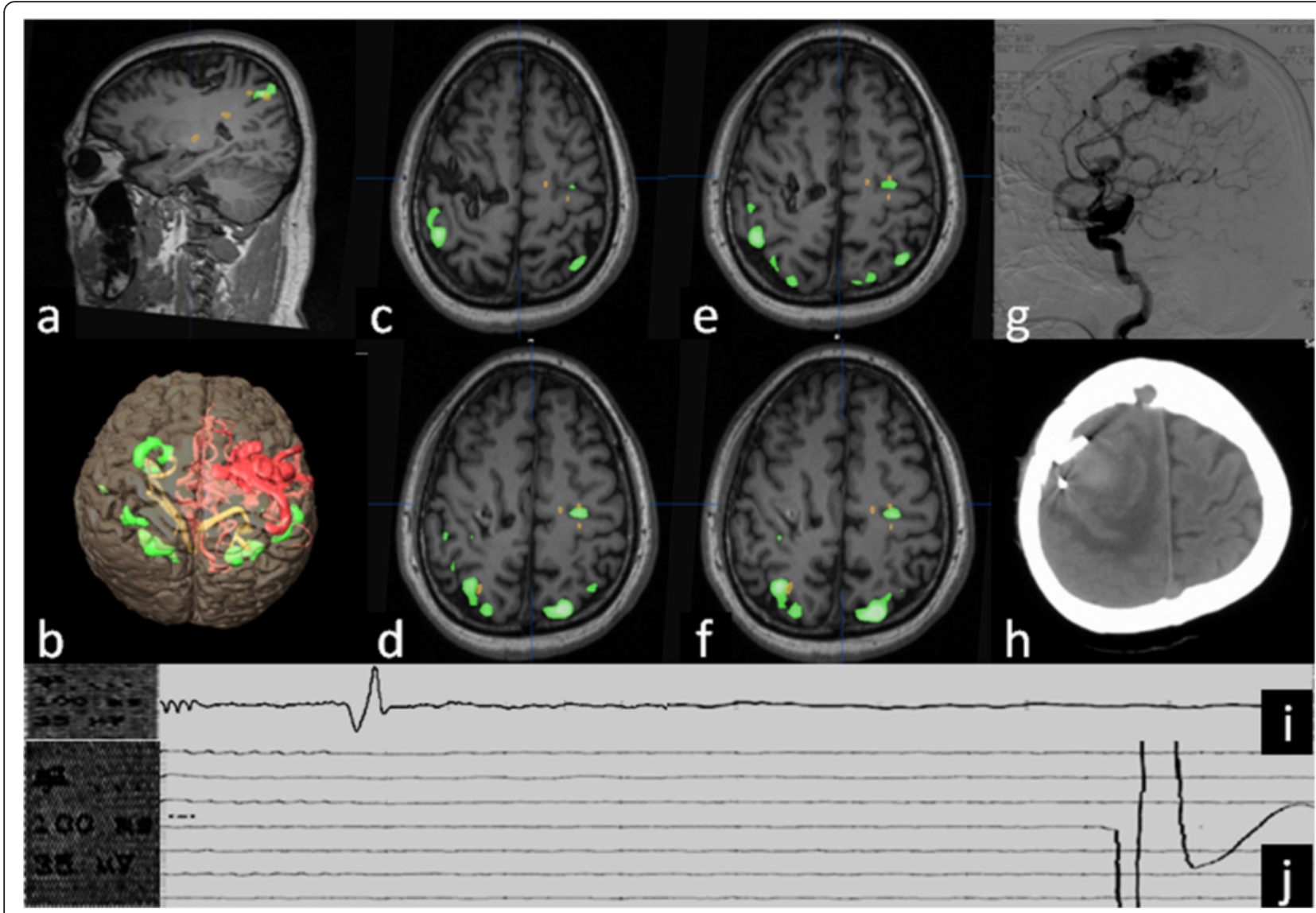

Fig. 1 a-g The AVM occupied the right percentral knob in patient 4. Left hand movement elicited the SA, SPL in the ipsilesional hemisphere and AMA, SPL in the contralesional hemisphere (in green). Two aberrant fiber tracts were tracked using the motor activation areas as the ROI (in yellow). One derived from ipsilesional SPL, and the other one derived from controlesional AMA. $\mathbf{h}$ Postoperative CT scan shows surgical field hemorrhage and surrounding edema. $\mathbf{i}$ and $\mathbf{j}$ Intraoperative electrical stimulation results showed that the positive stimulation sites were found at cortex (i) and subcortex levels (j) just behind the lesion

contralateral fiber tract was tracked even the peak motor activity point was located at cotralesional PMA (Fig. 5).

All the patients suffered from postoperative motor deficits, but the long-term prognoses were acceptable except in patient 1 (Table 1). Patient 1 suffered from postoperative cerebral hemorrhage, which caused a serious deterioration of motor functions of left limbs from grade III to I on the third postoperative day. He got a sudden onset of agitation, loss of consciousness and died on the 20th day due to the pulmonary embolism, caused by deep venous thrombosis. Patient 4 and patient 5 suffered from persistent motor deficits. Patient 4 showed complete weakness of the affected upper extremity postoperatively (grade I). She underwent postoperative rehabilitation and at 6month follow-up her right upper limb power had increased from grade I to IV. For patient 5 suffered from postoperative left hand motor deficits (grade III). The muscle strength of left hand recovered slowly to grade IV in 3 months after surgery, but without any progresses in the next 3 months. Patient 2, 3 had relatively good prognosis. As mentioned above, patient 3 postoperative muscle strength of right foot was IV, and that quickly recovered to an extent to nearly normal on the 7 th day after surgery. In patient 2, suffered transient motor deficit with grade III muscle strength of right hand but without persistent motor deficit in the 6 months followup period.

\section{Discussion}

Many studies have demonstrated that cAVMs involving motor cortex can cause different kinds of BOLD activations, elicited by contralateral hand or foot movements, which are considered as the plasticity of motor cortex [1, 2, 6-9]. In this study, the patients exhibited all the three kinds of motor cortex reorganization proposed by Alkadhi et al. [1], including activation in contralateral PMA in patient 5 , activation in secondary motor areas in patient 3,1 and 5, activation in affected PMA in patient 2, 3, 4 . 


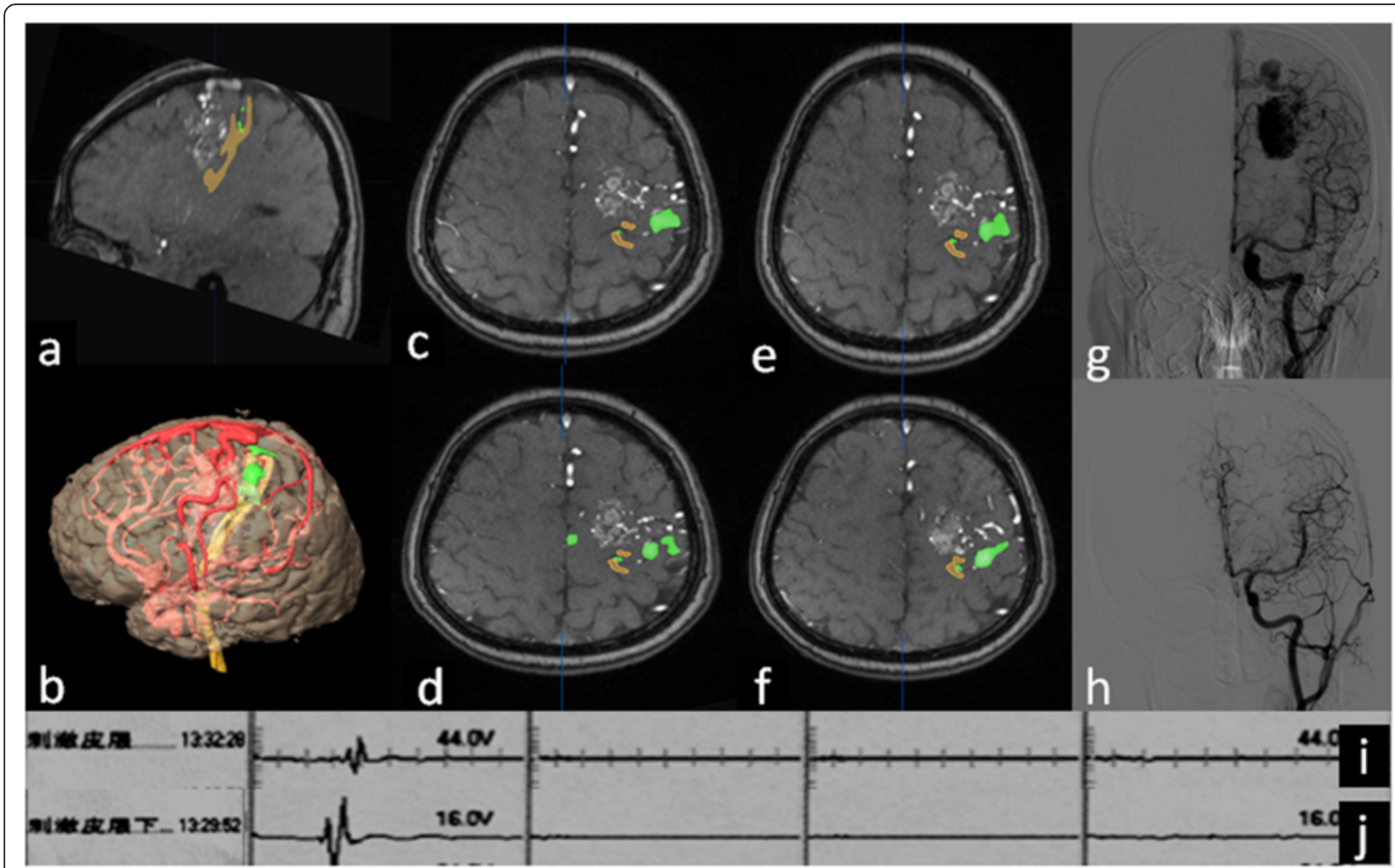

Fig. 2 a-g In patient 1, a compact AVM partially involved the left precentral knob. The residual precentral knob and SA were activated during right hand movement (in green). Functionally tracked fiber tract was behind the lesion and located in a normal anatomic pathway (in yellow). $\mathbf{h}$ Postoperative DSA shows radical resection. $\mathbf{i}$ and $\mathbf{j}$ Intraoperative electrical positive stimulation sites were found at cortex (i) and subcortex levels (j) just behind the lesion

Management of AVMs includes resection, endovascular embolization, radiosurgery, or a combination of these modalities. In the literature, conservative follow-up or radiosurgery are often recommended for patients with AVMs in or close to eloquent motor areas. At present, there is no standard guideline for the case selection in patients with motor strip AVM. Furthermore, Stereotactic radiosurgery (SRS), focused irradiation, can be effective for malformations that are smaller than $3 \mathrm{~cm}$, but complete obliteration requires approximately 1 to 3 years after treatment and cure is not always obtained. Delayed complications such as hemorrhage in the latency period and radiation edema or necrosis can occur as late complications. Therefore, in our hospital, the selection of the surgical or other treatments based on the clinical presentation, patient condition, patient required and prospective surgical risks. Surgery was only recommended for patients with serious headache, intractable seizure, progressive neurological deficit and previous hemorrhage.

All the patients suffered from postoperative motor deficits regardless of BOLD activation patterns. In patient 1 , fMRI showed activation areas were far from the lesion, but intraoperative ECS found cortical and subcortical positive stimulation points just around the lesion, also the patient suffered from motor deficits postoperatively. In patient 5, movements of left hand activated more voxels in the left hemisphere than that in contralateral hemisphere. Even the peak motor activity point was in the left PMA. However, the patient still suffered transient and persistent motor deficit postoperatively. It means that cortical reorganization performance on fMRI has no effect on postoperative motor function. As concluded by Ulmer JL and his colleagues [10], several features of fMRI should be considered when assessing a patient with apparent lesion-induced cortical reorganization: 1) cerebral lesions may induce neurovascular uncoupling, 2) decreased BOLD activity in eloquent cortex could be attributable to neuronal compromise and/or neurovascular uncoupling, 3) increased activity in uninvolved brain may indicate functional adaptation, but not necessarily cortical reorganization. From another perspective, the activated non-PMA areas may replace part of motor cortex? This may be important to maintain and support the normal motor function. The recent advances in fMRI and DTI, which enable 3-dimensional reconstruction of the major descending pathways and proper identification of 


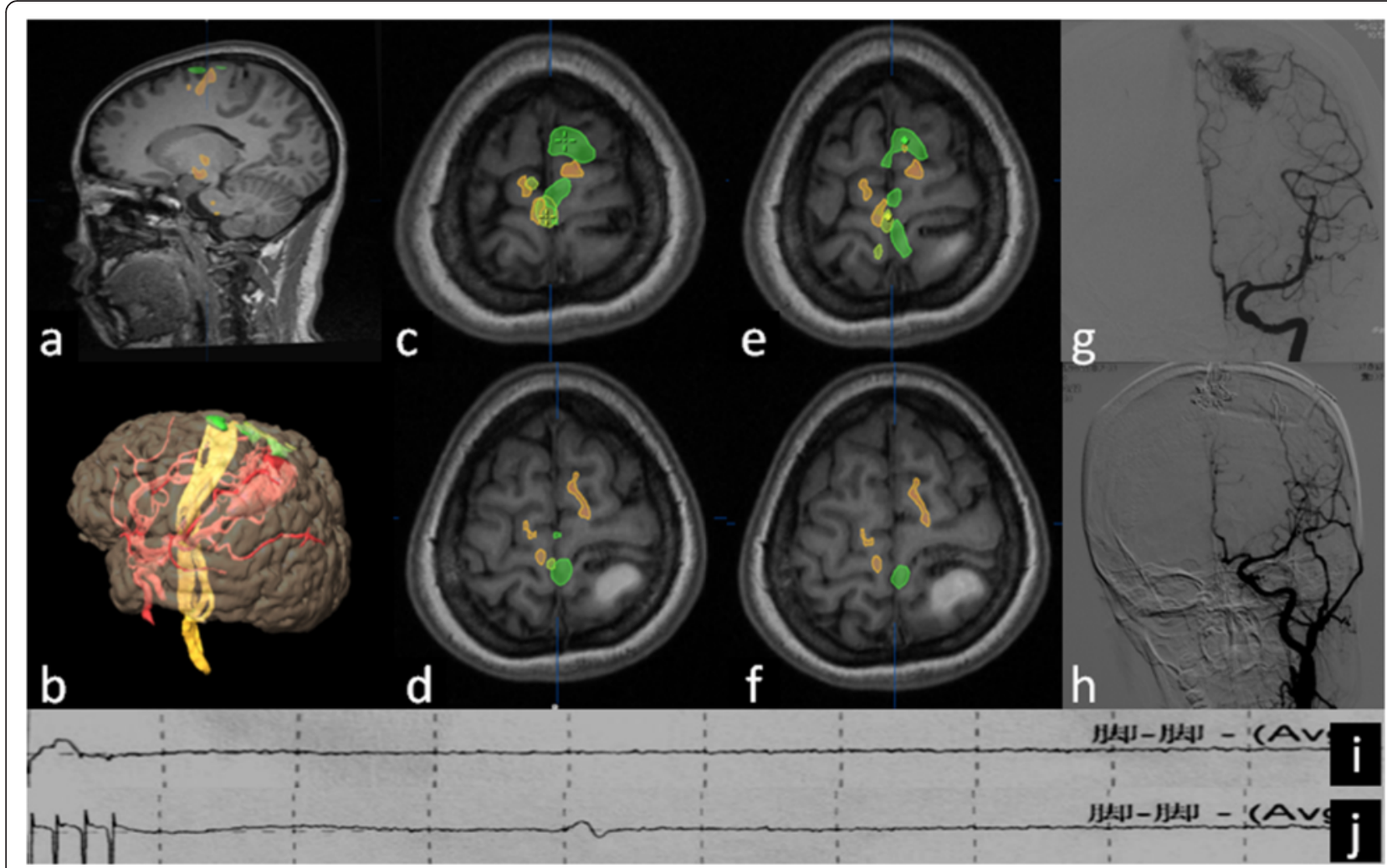

Fig. 3 a-g In patient 2, the lesion occupied the posterior part of left paracentral lobule. The activation areas elicited by right foot movements reorganized obviously at the anterior part of the paracentral lobule and SMA (in green) compared with that elicited by left foot movements in contralesional hemisphere (in paille). The functionally seeded CST fiber tract in ipsilesional hemisphere originated from anterior part of the paracentral lobule and SMA then traveled backwards to the anatomical CST pathway (in yellow), where the fiber was close to the lesion. $\mathbf{h}$ Postoperative DSA shows radical resection. $\mathbf{i}$ and $\mathbf{j}$ No cortical positive stimulation point around the lesion (i) and low amplitude subcortical positive stimulation points before the lesion ( $\mathbf{j}$ )

the motor cortex, have contributed significantly to reducing postoperative morbidity in recent years. The other hand, the unique features of AVMs, i.e., their hypervascularization and large vessels, pose a different challenge towards functional mapping. Therefore, surgical indications of AVM are comprehensive aspects. They couldn't be used to evaluate the motor function independently when the cortex surrounding the AVMs. So combined with the intraoperative navigation system and the valuable information gained from electrophysiological monitoring and direct brain stimulation.intraoperative ECS is necessary to protect motor function $[11,12]$.

CST is an important component of the motor system for controlling fine movements. Many studies have shown that damaged and reorganized CST associated clinical symptoms in patients with AVM involving motor areas [3, 13-16]. CAVMs are believed to form at approximately 3 weeks of gestation prior to brain specialization $[7,17]$, and the plasticity of fiber tracts might also happens following cortical reorganization in cAVMs patients. In this study, preoperative DTI showed that in 3 patients (patient2, 4 and 5) the CST were in (patient4) or around the AVMs and consistent with anatomical descriptions. These findings were validated by intraoperative ECS. Postoperatively patients 4 suffered from persistent motor deficits. Two of the five patients had functionally seeded CST far from the lesions. Patient 1 had two aberrant fiber tracts in preoperative functional mapping, which could not be validated by intraoperative electrical subcortical stimulation. But postoperative motor deficits and dramatically motor deterioration caused by postoperative local hemorrhage (Fig. 1), it indicated no motor function of the tracked aberrant fiber tracts. These aberrant fiber tracts could be technical artifacts of DTI tractography or the fiber tracts could exist but had no motor function $[18,19]$. Conversely, in patient 3motor activation area and CST fiber tract reorganized at SMA and anterior part of paracentral lobule, which is conserved the foot motor area, is consistent with ECS results. To conclude, during AVM surgery, the nidus should be resected radical 


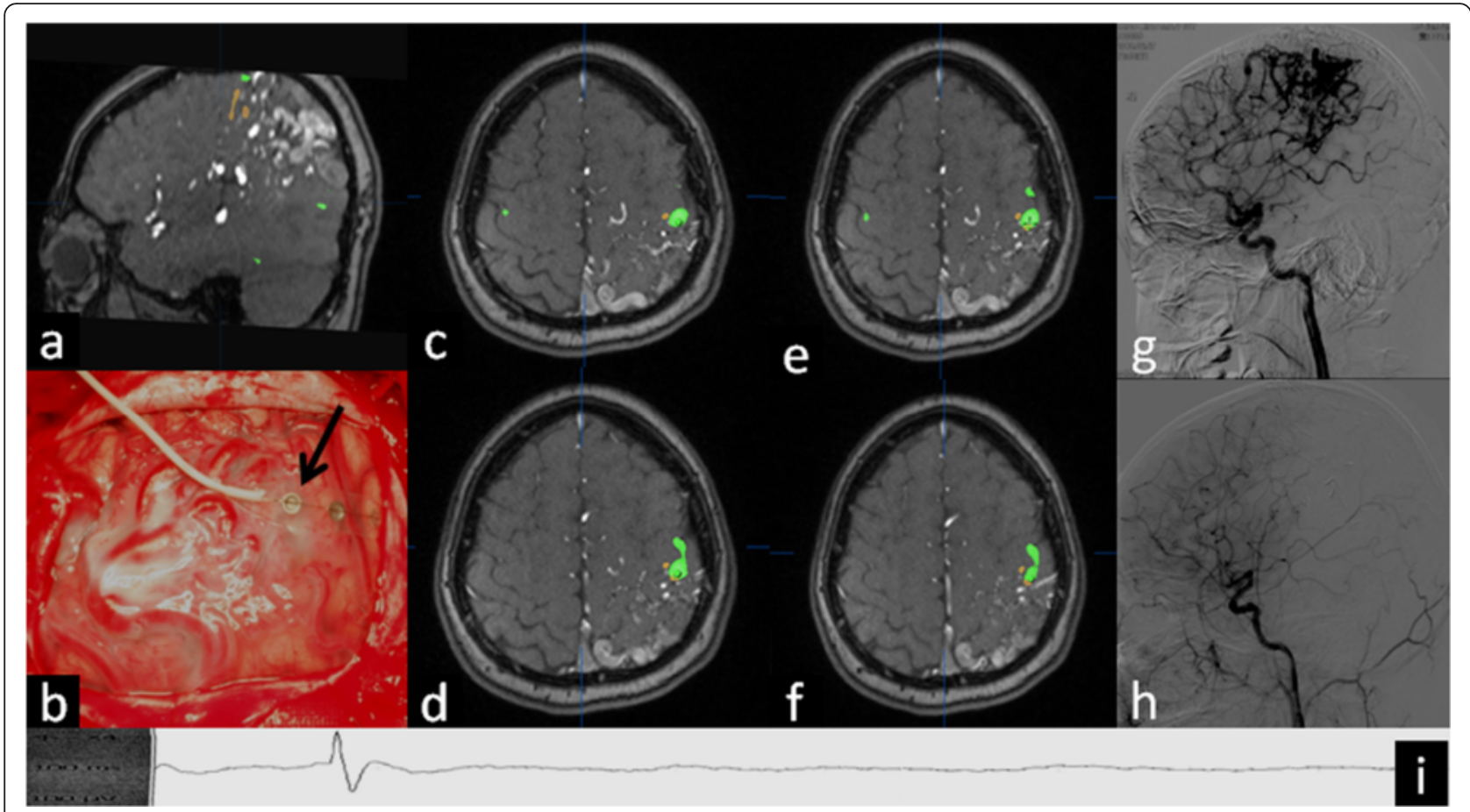

Fig. 4 a,c-g Patient 3 had a diffuse nidus of AVM, which involving left superior parietal lobule and precentral knob area. The only activation area elicited by right hand movement was within the nidus (in green). The CST fiber tract originated from the activation area and then passed through the nidus (in yellow). $\mathbf{h}$ Postoperative DSA shows radical resection. $\mathbf{b}$ and $\mathbf{i}$ Cortical positive simulation points were found at the brain parenchyma within the diffuse nidus intraoperatively (black arrow)

for residuals would lead to coagulation difficult and postoperative hemorrhage. So surgery of AVM is "all or none". At present, we do not suggest patient select surgery, who's CST through nidus before preoperative fMRI. Caution should be taken in the interpretation of aberrant fiber tracts. Moreover, we still need intraoperative electrical stimulation to verify the aberrant findings.

Prior studies about brain plasticity following perinatal brain injury and adult stroke have show many kinds of CST plasticity forms, including: 1) recovery of a damaged CST; 2) subcortical peri-lesional reorganization; 3) ipsilateral pathway from the contralesional hemisphere; 4) contribution of the secondary motor areas [20-22]. These studies also concluded that ipsilateral projection is associated with poor motor function, and it is a kind of maladaptive plasticity [23, 24]. This conclusion is consistent with our results. The fiber plasticity happened in the ipsilesional hemisphere at SMA (patient 2) or SA (patient 1 and 5), but none of the patient showed ipsilateral projection from contralateral hemisphere to contralateral limbs, which had normal preoperative motor functions except the aberrant fiber tracts in patent1. There were also activation areas in other areas but no fiber tract derived from them in patient 2,3 and 5 . We cannot give an explanation for these activation areas, so we can only provide some speculations for this finding. The central nervous system is an integrated, wide, plastic network, which is made up of cortical functional epicenters, and is connected by both short and large-scale white matter fiber tracts. The two motor cortices do not operate in isolation. Complex cortical motor circuits, both within and between the hemispheres, also mediate neuroplasticity [23]. DTI tractography fails to depict synchronization of eloquent cortical epicenters connected through white matter pathways [18] due to technical limitation. Further studies are still need to explore the underlying mechanism. In conclusion, the plasticity of motor fiber on preoperative DTI provides some useful indications for surgical outcomes, but ECS is still needed in surgery to confirm the preoperative findings.

\section{Limitation}

We caution that our sample was relatively small and no statistics in the paper, therefore, this study may have lacked sufficient power. Furthermore, in our medical conditions, postoperative follow-up is difficult for patient's compliance, most patients' follow-up only to telephone. So, it is regrettable that seldom patients to select reexamination fMRI. 


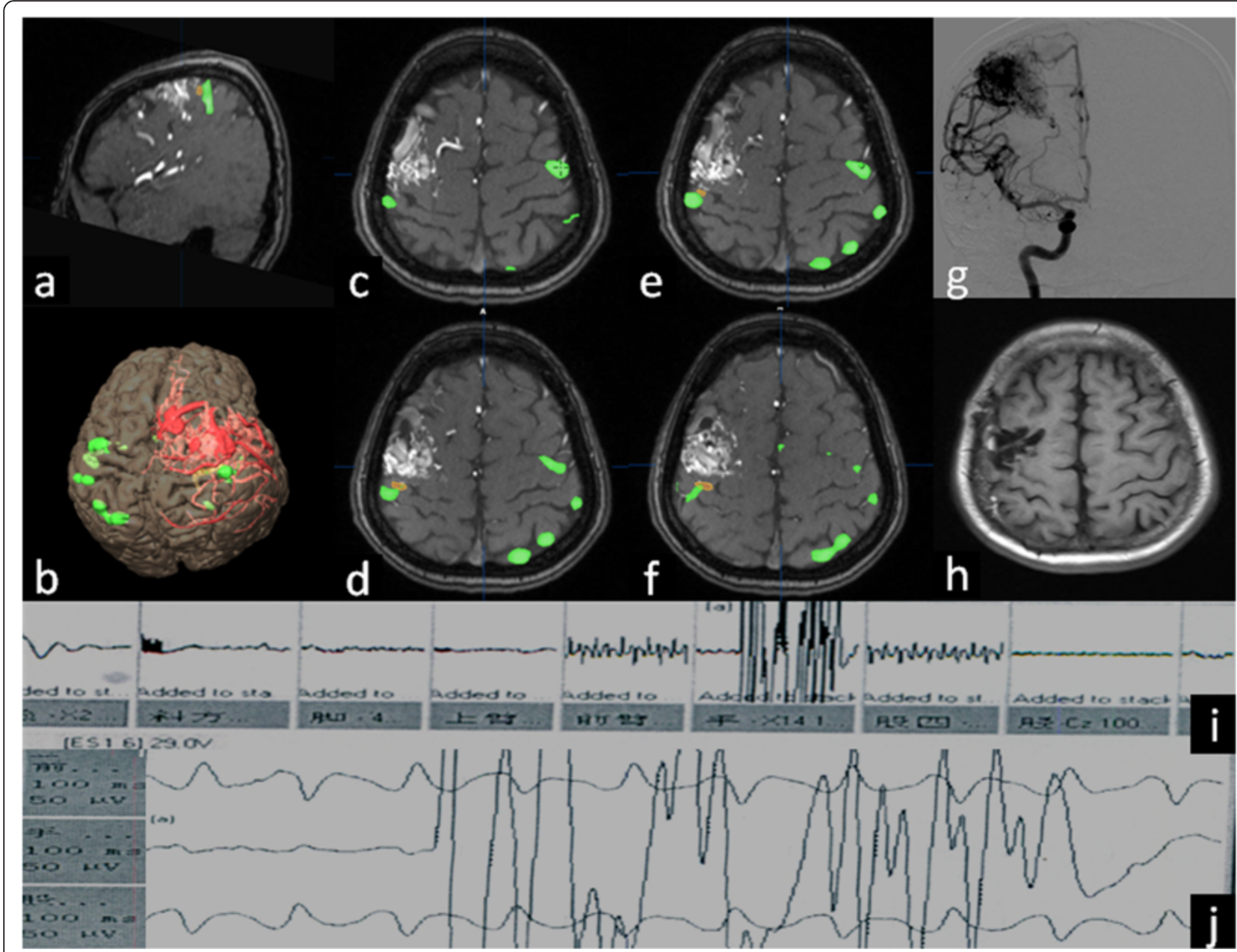

Fig. 5 a-g The AVM involving the right precentral knob in patient 5. Ipsilesional SA, contralesional SPL and contralesional PMA were activated by left hand movement (in green). The peak motor activity point was located at cotralesional PMA (green start). Functionally tracked fiber tracts derived from ipsilesional SA and travelled along the anatomic CST pathways (in yellow). $\mathbf{h}$ Postoperative MRI scan. $\mathbf{i}$ and $\mathbf{j}$ Intraoperative electrical positive stimulation sites were found at cortex (i) and subcortex levels (j) just behind the lesion

\section{Conclusion}

The plasticity of motor fiber on preoperative DTI provides some useful indications for surgical outcomes, but ECS is still needed in surgery to confirm the preoperative findings.

\section{Competing interests}

The authors declare that they have no competing interests.

\section{Authors' contributions}

LWW and FXL collected the clinical information from the database and manscript writing. The anatomic locations of the activation and peak motor activity point for each paradigm were documented by WJ and YMJ. YLZ and $Y C$ manage to revise manscript. The operations were performed by the experienced vascular neurosurgeon SW. All authors read and approved the final manuscript.

\section{Disclosure}

This study was supported by a "National science and technology Support Plan" (No.2011BAl08B08, Principle investigator: Professor Shuo Wang), grant from the Ministry of Health of China. The authors declare that they have no conflict of interest.

\section{Author details}

'Department of Neurosurgery, Beijing Tiantan Hospital, Capital Medical University, Beijing, P. R. China. ${ }^{2}$ Department of Neurosurgery, Hongqi Hospital, Mudanjiang Medical University, Aimin District, Mudanjiang, Heilongjiang Province, P. R. China.

Received: 14 July 2015 Accepted: 8 December 2015

Published online: 25 April 2016

\section{References}

1. Alkadhi H, Crelier GR, Golay X, et al. Plasticity of the human motor cortex in patients with arteriovenous malformations: a functional MR imaging study. AJNR Am J Neuroradiol. 2000;21 (8):1423-33. PMID: 11003274 [PubMed - indexed for MEDLINE].

2. Lee $\mathrm{L}$, Sitoh $\mathrm{YY}, \mathrm{Ng}$ l, et al. Cortical reorganization of motor functional areas in cerebral arteriovenous malformations. J Clin Neurosci. 2013;20(5):649-53.

3. Ellis MJ, Rutka JT, Kulkarni AV, et al. Corticospinal tract mapping in children with ruptured arteriovenous malformations using functionally guided diffusion-tensor imaging. J Neurosurg Pediatr. 2012;9(5):505-10.

4. Jang SH. A review of diffusion tensor imaging studies on motor recovery mechanisms in stroke patients. NeuroRehabilitation. 2011;28(4):345-52. PMID: 21725167 [PubMed - indexed for MEDLINE]. 
5. Scantlebury N, Gaetz W, Widjaja E, et al. Functional reorganization of the corticospinal tract in a pediatric patient with an arteriovenous malformation. Neuroreport. 2014;25(1):55-9.

6. Atlas SW, Howard RN, Maldjian J, et al. Functional magnetic resonance imaging of regional brain activity in patients with intracerebral gliomas: findings and implications for clinical management. Neurosurgery. 1996; 38(2):329-38.

7. Baciu M, Le Bas JF, Segebarth C, et al. Presurgical fMRI evaluation of cerebral reorganization and motor deficit in patients with tumors and vascular malformations. Eur J Radiol. 2003;46(2):139-46.

8. Carpentier AC, Constable RT, Schlosser MJ, et al. Patterns of functional magnetic resonance imaging activation in association with structural lesions in the rolandic region: a classification system. J Neurosurg. 2001;94(6):946-54.

9. Ulmer JL, Hacein-Bey L, Mathews VP, et al. Lesion-induced pseudo-dominance at functional magnetic resonance imaging: implications for preoperative assessments. Neurosurgery. 2004;55(3):569-79. discussion 580-1.

10. Itoh $\mathrm{D}$, Aoki S, Maruyama K, et al. Corticospinal tracts by diffusion tensor tractography in patients with arteriovenous malformations. J Comput Assist Tomogr. 2006;30(4):618-23.

11. Gabarrós A, Young WL, McDermott MW, Lawton MT. Language and motor mapping during resection of brain arteriovenous malformations: indications, feasibility, and utility. Neurosurgery. 2011;68:744-52.

12. Lepski G, Honegger J, Liebsch $\mathrm{M}$, et al. Safe resection of arteriovenous malformations in eloquent motor areas aided by functional imaging and intraoperative monitoring. Neurosurgery. 2012;70:276-88.

13. Lazar M, Alexander AL, Thottakara PJ, et al. White matter reorganization after surgical resection of brain tumors and vascular malformations. AJNR Am J Neuroradiol. 2006;27(6):1258-71. PMID: 16775277 [PubMed - indexed for MEDLINE].

14. Okada T, Miki Y, Kikuta K, et al. Diffusion tensor fiber tractography for arteriovenous malformations: quantitative analyses to evaluate the corticospinal tract and optic radiation. AJNR Am J Neuroradiol. 2007;28(6): 1107-13.

15. Yamada K, Kizu O, Ito H, et al. Tractography for arteriovenous malformations near the sensorimotor cortices. AJNR Am J Neuroradiol. 2005;26(3):598-602. PMID: 15760872 [PubMed - indexed for MEDLINE].

16. Moftakhar P, Hauptman JS, Malkasian D, et al. Cerebral arteriovenous malformations. Part 2: physiology. Neurosurg Focus. 2009;26(5):E11.

17. Duffau H. Diffusion tensor imaging is a research and educational tool, but not yet a clinical tool. World Neurosurg. 2014;82(1-2):e43-5.

18. Duffau $\mathrm{H}$. The dangers of magnetic resonance imaging diffusion tensor tractography in brain surgery. World Neurosurg. 2014;81(1):56-8.

19. George E, Heier L, Kovanlikaya I, et al. Diffusion tensor imaging of pyramidal tract reorganization after pediatric stroke. Childs Nerv Syst. 2014;30(6):1135-9.

20. Hallett M. Plasticity of the human motor cortex and recovery from stroke. Brain Res Brain Res Rev. 2001;36(2-3):169-74.

21. Jang SH, Hong JH, Ahn SH, et al. Motor function reorganization in a patient with a brainstem lesion: DTT, fMRI and TMS study. NeuroRehabilitation. 2010;26(2):167-71. PMID: 20203384 [PubMed - indexed for MEDLINE].

22. Kirton A. Modeling developmental plasticity after perinatal stroke: defining central therapeutic targets in cerebral palsy. Pediatr Neurol. 2013;48(2):81-94.

23. Staudt M. Brain plasticity following early life brain injury: insights from neuroimaging. Semin Perinatol. 2010;34(1):87-92.

24. De Benedictis A, Duffau H. Brain hodotopy: from esoteric concept to practical surgical applications. Neurosurgery. 2011;68(6):1709-23. discussion 1723. PMID: 21346655 [PubMed - indexed for MEDLINE].

\section{Submit your next manuscript to BioMed Central and we will help you at every step:}

- We accept pre-submission inquiries

- Our selector tool helps you to find the most relevant journal

- We provide round the clock customer support

- Convenient online submission

- Thorough peer review

- Inclusion in PubMed and all major indexing services

- Maximum visibility for your research

Submit your manuscript at www.biomedcentral.com/submit
Biomed Central 